NASA Technical Memorandum 87093

\title{
Forced Response Analysis of an Aerodynamically Detuned Super- sonic Turbomachine Rotor
}

\begin{tabular}{|c|c|c|c|c|c|c|}
\hline (NASA-TH-87093) & FORCED RE & ESPONSE & ANAIYSIS & OF & & N $86-10019$ \\
\hline AN AERODYNAMICAIIYY & Y DETUNEI & D SUPERS & ONIC & & & \\
\hline TORBOHACHINE ROTOR & $\mathrm{R}$ (NASA) & $24 p$ & & & & \\
\hline HC AO2/ME A01 & & & CSCL & $01 \mathrm{~A}$ & $G 3 / 02$ & $\begin{array}{l}\text { Onclas } \\
27534\end{array}$ \\
\hline
\end{tabular}

Daniel Hoyniak

Lewis Research Center

Cleveland, Ohio

and

Sanford Fleeter

Purdue University

West Lafayette, Indiana

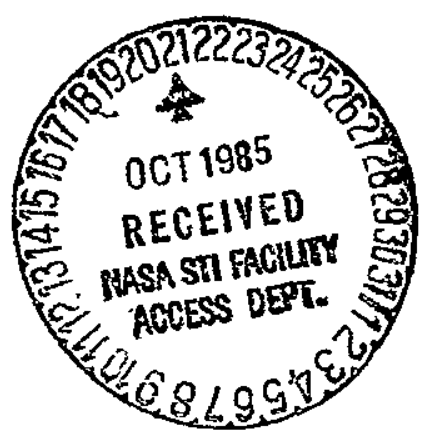

Prepared for the

10th Biennial Design Engineering Division Conference and Exhibit on Mechanical Vibration and Noise

sponsored by the American Society of Mechanical Engineers

Cincinnati, Ohio, September 10-13, 1985 
FORCED-RESPONSE ANALYSIS OF AN AERODYNAMICALLY DETUNED

SUPERSONIC TURBOMACHINE ROTOR

\author{
Daniel Hoyniak \\ National Aeronautics. and Space Administration \\ Lewis Research Center \\ Cleveland, Ohio 44735 \\ and \\ Sanford Fleeter \\ Purdue University \\ School of Mechanical Engineering \\ West Lafayette, Indiana

\section{SUMMARY}

High-performance aircraft-engine fan and compressor blades are vulnerable to aerodynamically forced vibrations generated by inlet flow distortions due to wakes from upstream blade and vane rows, atmospheric gusts, and maldistributions in inlet ducts. In this report, an analysis is developed to predict the flow-induced forced response of an aerodynamically detuned rotor operating in a supersonic flow with a subsonic axial component. The aerodynamic detuning is achieved by alternating the circumferential spacing of adjacent rotor blades. The total unsteady aerodynamic loading acting on the blading, as a result of the convection of the transverse gust past the airfoil cascade and the resulting motion of the cascade, is developed in terms of influence coefficients. This analysis is used to investigate the effect of aerodynamic detuning on the forced response of a 12-blade rotor; with Verdon's Cascade B flow geometry as a uniformly spaced baseline configuration. The results of this study indicate that, for forward-traveling wave gust excitations, aerodynamic detuning is very beneficial, resulting in significantly decreased maximum-amplitude blade responses for many interblade phase angles.

\title{
INTRODUCTION
}

The blades of high-performance aircraft engines are thin, and their outer span regions operate in a high Mach number flow regime. As a result, the blade rows are susceptible to aerodynamically induced forced vibrations in the supersonic inlet flow regime, generated by inlet flow distortions due to wakes from upstream blade and vane rows, atmospheric gusts, and maldistributions in inlet ducts.

To predict the aerodynamically induced forced response and stability of a conventional blade row, aeroelastic analyses are used which consider the individual blades to be identical, that is, to have the same mass and stiffness. In fact, individual rotor blades are not identical; they have varying ranges of natural frequencies due to manufacturing tolerances.

Whitehead (ref. 1) was the first to discuss the effect that such random airfoil-to-airfoil variations in natural frequency, defined as structural mistuning, have on the aeroelastic characteristics of a rotor. Recentiy, several 
investigators (refs. 2 to 4 ), have considered the deliberate introduction of blade-to-blade variations in natural frequencies, defined as structural detuning, as a passive aeroelastic control mechanism. These analyses show that structural detuning provides a substantial improvement in aeroelastic stability. However, structural detuning is not universally accepted as a viable passive flutter control mechanism because of its associated manufacturing, material, inventory, engine maintenance, contro1, and cost problems (ref. 5).

A new approach to passive aeroelastic control, developed in reference 6 with regard to supersonic unstalled torsional flutter, is aerodynamic detuning, defined as passage-to-passage variations in the unsteady aerodynamic flow field of a blade row. Thus, aerodynamic detuning results in blade-to-blade differences in the unsteady aerodynamics acting on the individual blades of a rotor. Consequently, the blades do not respond in a classical traveling wave mode typical of the aeroelastic behavior of a conventional aerodynamically tuned rotor.

In this report, the analysis developed in reference 6 is extended in order to predict the flow-induced forced response of an aerodynamically detuned rotor operating in a supersonic flow with a subsonic axial component. The rotor is modeled as a flat plate airfoil cascade, representing an unwrapped rotor annulus. The aerodynamic detuning is achieved by alternating the circumferential spacing of adjacent rotor blades, as described in reference 6 . The total unsteady aerodynamic loading on the blades, due to the convection of the transverse gust past the airfoil cascade as well as the motion of the cascade, is developed in terms of influence coefficients. This technique makes it possible to consider various gust loadings without having to perform a complete unsteady aerodynamic analys is for each interblade phase angle. This analys is is used to investigate the effect of aerodynamic detuning on the flow-induced forced response of a 12-blade rotor, with Verdon's Cascade B flow geometry (ref. 7) as a uniformly spaced baseline configuration.

\section{SYMBOLS}

c perturbation sonic velocity

$c_{M}^{n}$ influence coefficient of airfoil, $n$

c airfoil chord

g structural damping coefficient

I mass moment of inertia

K spring constant

$k$ reduced frequency, $k=\omega c / u_{\infty}$

M dimensionless unsteady aerodynamic moment

$M_{\infty}$ cascade inlet Mach number

$S$ uniform airfoil spacing 




Subscripts:

d detuned cascade

g gust

n airfoil number

R reference airfoil uniformly spaced cascade

$R_{e}$ reference for set of even numbered airfoils

$R_{0}$ reference for set of odd numbered airfoils

\section{Superscripts:}

g gust

m motion dependent

UNSTEADY AERODYNAMIC MODEL

The aeroelastic analysis of conventional tuned and structurally detuned blade rows uses two-dimensional, unsteady aerodynamic models applied in a strip theory technique. Hence, a two-dimensional, equally spaced airfoil cascade is used to represent a typical element of the blade row. In the high Mach number flow regime of significance to turbomachines, a flat plate airfoil cascade 
embedded in a supersonic flow field with a subsonic leading edge locus is considered (fig. 1).

The total unsteady aerodynamic blade loading for the forced-response problem is determined from two separate models: (1) a transverse-gust model for the inlet distortion and (2) a motion-dependent model for the resulting vibratory response. The gust loading is determined by considering a sinusoidal transverse gust being swept past the stationary cascade. The motion-dependent unsteady aerodynamics are obtained by considering the resulting torsion mode harmonic oscillations of the cascade. These two models are then combined to predict the total unsteady aerodynamic airfoil loading in a classical traveling wave mode, that is, with a constant interblade phase angle $\beta$ between adjacent airfoils.

The fluid is assumed to be an inviscid, perfect gas with the flow isentropic, adiabatic, and irrotational. The unsteady continuity and Euler equations are linearized by assuming that the unsteady perturbations are small as compared with the uniform through flow. Thus, in the motion-dependent unsteady aerodynamic model, the boundary conditions, which require that the unsteady flow be tangent to the airfoil surfaces and that the normal velocity be continuous across the wakes, are applied on the mean positions of the oscillating airfoils. The flow tangency condition of the transverse-gust model is applied on the airfoil positions as this model uses a stationary airfoil cascade.

The unsteady cascade aerodynamics and, in particular, the unsteady aerodynamic forces and moments acting on the uniformly spaced airfoils can be predicted by using various techniques. Of particular interest are the analyses of Verdon (ref. 8), Brix and Platzer (ref. 9), and Caruthers and Riffel (ref. 10); these analyses use a finite cascade representation of a semi-infinite cascade. The cascade periodicity condition is enforced by stacking sufficient numbers of uniformly spaced single airfoils until convergence in the unsteady flow field is achieved.

For the aerodynamically detuned, alternate circumferentially spaced blade row, an analogous unsteady aerodynamic model is developed. A finite cascade representation of the aerodynamically detuned blade row is shown in figure 2 . There are two distinct flow passages: (1) a reduced spacing, or increased solidity, passage and (2) an increased spacing, or reduced solidity, passage. This design results in two distinct sets of airfoils which are defined as the even- and odd-numbered sets of airfoils. Thus, for this aerodynamically detuned cascade, two-passage periodicity is required; that is, the periodic cascade, unsteady flow field is achieved by stacking sufficient numbers of two alternately spaced flow passages or two airfoils, one from each set.

The formulation of the linearized differential equations describing the unsteady perturbation flow field for the finite aerodynamically detuned cascade is based on the method of characteristics analysis of the finite uniformiy spaced airfoil cascade of Brix and Platzer (ref. 9). In particular the dependent variables are the nondimensionalized chordwise, norma1, and sonic perturbation velocities, $U, V$, and $C$, respectively. The independent variables are the dimensionless chordwise, normal coordinates $x$ and $y$ and time $t$. When harmonic time dependence at a frequency $\omega$ is assumed, differential equations describing the unsteady perturbation flow field result: 


$$
\left.\begin{array}{c}
\frac{\partial U}{\partial x}+\sqrt{M_{\infty}^{2}-1} \frac{\partial V}{\partial y}+\frac{\partial C}{\partial x}+i k M_{\infty}^{2} C=0 \\
\frac{\partial U}{\partial x}+\frac{\partial C}{\partial y}+i k U=0 \\
\frac{\partial U}{\partial y}-\sqrt{M_{\infty}^{2}-1} \frac{\partial V}{\partial x}=0
\end{array}\right\}
$$

The boundary conditions for the gust model are developed by considering a small transverse gust of amplitude $W_{g}$ superimposed on and convected with the uniform through flow with a mean velocity $u_{\infty}$ past the stationary aerodynamically detuned cascade. The airfoil-surface flow tangency condition requires that the normal perturbation velocity be equal and opposite to the velocity induced by the passage of the gust. Thus, as indicated in reference 11 , the nondimensional normal perturbation velocity on the airfoils due to the gust is specified by

$$
v^{g}\left(x, y_{s}, t\right)=\frac{w_{g}}{u_{\infty}} e^{i k(t-x)}
$$

where the superscript $g$ denotes gust, $x$ is the nondimensional airfoil chordwise coordinate as measured from midchord, $k=\omega c / u_{\infty}$ is the reduced frequency with $\omega$ the gust frequency, and $y_{s}$ denotes the airfoil surface.

To account for the response of the airfoil cascade to the gust, the. motion-dependent boundary conditions for the aerodynamically detuned cascade must also be specified. According to reference 6 , the normal perturbation velocity boundary condition for an airfoil cascade executing harmonic torsional oscillations about an elastic axis located at $x_{0}$ as measured from the leading edge is specified by

$$
v^{m}\left(x, y_{s}, t\right)=-\alpha\left[1+\left(x-x_{0}\right) i k\right] e^{i k t}
$$

where the superscript $m$ denotes motion dependence, and $\alpha$ is the amplitude of oscillation.

In this model, the unsteady aerodynamic loading is calculated by using an influence coefficient technique, as discussed in the next section. Hence, no interblade phase angle is specified in these boundary conditions (eqs. (2) and (3)).

\section{AERODYNAMIC INFLUENCE COEFFICIENTS}

The unsteady aerodynamic loading on the aerodynamically detuned cascade, composed. of the gust and the motion-dependent unsteady aerodynamics, is determined by using an influence coefficient technique. The aerodynamic influence coefficients are calculated for a given cascade geometry, inlet Mach number, and reduced frequency. The gust and motion-dependent unsteady aerodynamics are then calculated for a specified interblade phase angle by using a vector summation of the influence coefficients. 
The unsteady aerodynamic moment on the reference airfoil of a tuned cascade $M_{R}$ is defined in terms of influence coefficients for the gust $M_{R}^{g}$ and for the resulting motion-dependent unsteady aerodynamics $M_{R}^{m}$ :

$$
\begin{aligned}
& M_{R}=M_{R}^{g}+M_{R}^{m}=\left\{W_{0}\left[c_{g}^{0}\right]_{R}+w_{1}\left[c_{g}^{l}\right]_{R}+\ldots+w_{R}\left[c_{g}^{R}\right]_{R}+\ldots+w_{N}\left[c_{g}^{N}\right]_{R}\right\} e^{i \omega t} \\
& +\left\{\hat{\alpha}_{0}\left[c_{m}^{0}\right]_{R}+\hat{\alpha}_{1}\left[c_{m}^{l}\right]_{R}+\ldots+\hat{\alpha}_{R}\left[c_{m}^{R}\right]_{R}+\ldots+\hat{\alpha}_{N}\left[c_{m}^{N}\right]\right\} e^{i \omega t}
\end{aligned}
$$

Here $w_{n}$ and $\alpha_{n}$ denote the complex amplitude of the normal perturbation gust velocity on airfoil $n$ and the amplitude of motion of airfoil $n$, respectively. The influence coefficients $\left|c_{g}^{n}\right|_{R}$ and $\left|c_{m}^{n}\right|_{R}$ represent the moment on. the reference airfoil $R$ due to a gust of unit amplitude acting on airfoil $n$ and unit amplitude motion of airfoil $n$, respectively.

The aerodynamically detuned, alternate circumferentially spaced airfoil cascade is composed of two distinct flow passages and two sets of airfoils, defined as the sets of even- and odd-numbered airfoils. Thus, two reference airfoils, $R_{e}$ and $R_{0}$, must be considered. The total unsteady aerodynamic. moment acting on these reference airfoils is written in terms of influence coefficients:

$$
\begin{aligned}
& M_{R_{e}, R_{0}}=\left\{M_{R_{e}, R_{0}}^{g}+M_{R_{e}}^{m}, R_{0}\right\}=\left\{W_{0}\left[c_{g}^{0}\right] R_{e}, R_{0}+W_{1}\left[c_{g}^{l}\right] R_{e}, R_{0}+\ldots\right. \\
& \left.+w_{R_{e}}\left[\begin{array}{c}
R_{g} \\
C^{2}
\end{array}\right] R_{e}, R_{0}+w_{R_{0}}\left[\begin{array}{c}
R_{0} \\
C_{g}
\end{array}\right] R_{e}, R_{0}+\ldots+w_{N}-\left[\begin{array}{l}
C^{N} \\
-g
\end{array}\right] R_{e}, R_{0}\right\} e^{i \omega t} \\
& +\left\{\hat{\alpha}_{0}\left[\begin{array}{c}
c_{m}^{0}
\end{array}\right] R_{e}, R_{0}+\hat{\alpha}_{1}\left[\begin{array}{l}
C_{m} \\
\alpha^{\prime}
\end{array}\right] R_{e}, R_{0}+\ldots+\hat{\alpha}_{R_{e}}\left[\begin{array}{c}
R_{e} \\
C_{m}
\end{array}\right] R_{e}, R_{0}\right. \\
& \left.+\hat{\alpha}_{R_{0}}\left[c_{m}^{R_{0}}\right] R_{e}, R_{0}+\ldots+\hat{\alpha}_{N}\left[c_{m}^{N}\right] R_{e}, R_{0}\right\} e^{i \omega t}
\end{aligned}
$$

where the double subscript notation is a shorthand means of expressing two equations, one for each of the reference airfoils $R_{e}$ and $R_{0}$.

The influence coefficients of equation (5) are obtained by calculating the unsteady aerodynamic moment on the reference airfoils $R_{e}$ and $R_{0}$ independentiy, subject to the boundary conditions for the gust and motiondependent unsteady aerodynamics, equations (2) and (3), as applied to the airfoil number $n$.

For a tuned cascade subject to an inlet gust, the interblade phase angle between two adjacent airfoils $\beta$ i.s a function of the airfoil spacing for a given harmonic (ref. 12). Thus, for the detuned cascade, the interblade phase 
angle between two adjacent odd- or even-numbered airfoils $B_{d}$ is a function of the airfoil spacing for a given harmonic of the inlet gust $W_{g}$. Hence, for the aerodynamically detuned cascade, the gust and the motion-dependent normal velocity perturbations on airfoil $n$ can be written in terms of the corresponding velocities on the reference airfoils $R_{e}$ and $R_{0}$ :

$$
v_{n}=\left\{w_{R_{e}, R_{0}}+\hat{\alpha}_{R_{e}}, R_{0}\right\} e^{i n \beta d}
$$

where $\beta_{d}$ is the constant interblade phase angle between adjacent even- or odd-numbered airfoils, considering the reference airfolls $R_{e}$ and $R_{0}$ as airfoils numbers 0 and 1 , respectively. Analogous expressions can be developed for other choices of the reference airfoils.

The substitution of equation (6) into equation (5) and the subsequent grouping of the sets of even-and odd-numbered airfoils lead to the following. expression for the total unsteady aerodynamic moment on the two reference airfoils:

$$
\begin{aligned}
M_{R_{e}, R_{0}}=\left\{W_{R_{e}}[C G] R_{e}, R_{0}+W_{R_{0}}\right. & {\left.[C G] \begin{array}{l}
1 \\
R_{e}, R_{0}
\end{array}\right\} e^{i \omega t} } \\
& +\left\{\hat{\alpha}_{R_{e}}[C M] \hat{R}_{e}, R_{0}+\hat{\alpha}_{R_{0}}[C M] R_{e}^{1}, R_{0}\right\} e^{i \omega t}
\end{aligned}
$$

where

$$
\begin{aligned}
& {[C G] R_{e}^{0}, R_{0}=\left[\begin{array}{l}
0 \\
g
\end{array}\right] R_{e}, R_{0}+e^{i \beta} d\left[\begin{array}{l}
2 \\
g
\end{array}\right] R_{e}, R_{0}+\ldots+e^{1\left[\frac{N-1}{2}\right] \beta_{d}}\left[C_{g}^{N-1}\right] R_{e}, R_{0}} \\
& {[C G]{ }_{R}^{1}, R_{0}=\left[\begin{array}{l}
l \\
g
\end{array}\right] R_{e}, R_{0}+e^{i \beta} d\left[\begin{array}{c}
3 \\
g
\end{array}\right] R_{e}, R_{0}+\ldots+e^{1\left[\frac{N-3}{2}\right] \beta} d\left[C_{g}^{N-2}\right] R_{e}, R_{0}} \\
& {[C M]{ }_{R_{e}, R_{0}}^{0}=\left[c_{m}^{0}\right] R_{e}, R_{0}+e^{i \beta d}\left[\begin{array}{c}
2 \\
m
\end{array}\right] R_{e}, R_{0}+\ldots+e^{1\left[\frac{N-1}{2}\right] \beta_{d}}\left[c_{m}^{N-1}\right] R_{e}, R_{0}} \\
& {[C M]{ }_{R_{e}}^{1}, R_{0}=\left[C_{m}^{l}\right] R_{e}, R_{0}+e^{i \beta} d\left[C_{m}^{3}\right] R_{e}, R_{0}+\ldots+e^{1\left[\frac{N-3}{2}\right] \beta_{d}}\left[C_{m}^{N-2}\right] R_{e}, R_{0}}
\end{aligned}
$$

and $N$ denotes the total number of airfoils in the cascade. Periodicity for the aerodynamically detuned cascade is achieved by stacking two airfoil passages at a time.

The terms $[C G]_{R_{e}}^{0}, R_{0}$ and $[C M]_{R_{e}}^{0}, R_{0}$ represent the unsteady aerodynamic moment on the two reference airfoils $R_{e}$ and $R_{0}$. This moment is due to a unit amplitude gust acting on the set of even-numbered airfoils and due to the 
unit amplitude motion of the set of even-numbered airfoils, respectively, with the interblade phase angle between the even-numbered airfoils specified by $\beta_{d}$. The terms $[C G]_{R_{e}}^{1}, R_{0}$ and $[C M]_{R_{e}}^{1}, R_{0}$ have an analogous physical interpretation in terms of the unsteady aerodynamic moment on the two reference airfoils that are associated with the set of odd-numbered airfoils.

The level of aerodynamic detuning $\varepsilon$ is defined as follows for the alternate circumferentially spaced airfoil cascade:

$$
S_{1,2}=(1 \mp \varepsilon) S
$$

where $S$ is the spacing of the uniformly spaced tuned cascade (fig. 1), and $S_{1}$ and $S_{2}$ denote the spacing of the reduced and increased flow passages of the aerodynamically detuned cascade (fig. 2). The relationship between the interblade phase angle for a uniformiy spaced cascade $\beta$ and the aerodynamically detuned cascade interblade phase angle $\beta_{d}$ is given by

$$
\beta_{d}=2 \beta
$$

The complex gust amplitudes $W_{R_{e}}$ and $W_{R_{0}}$ are related to the Fourier coefficients of a general inlet distortion and to the airfoil spacing. Thus, for a given harmonic of the inlet distortion, these amplitudes are related by the interblade phase angle between adjacent odd- and even-numbered airfoils $\beta_{d}$ and the level of aerodynamic detuning $\varepsilon$ :

$$
W_{R_{0}}=W_{R_{e}} e^{i(7-\varepsilon) \beta_{d} / 2}
$$

Thus, the total unsteady aerodynamic moment used in this analysis, obtained by substituting equation (10) into equation (7) is given by

$$
\begin{aligned}
\left.M_{R_{e}, R_{0}=W_{R_{e}}\left\{[C G] l_{e}^{0}, R_{0}\right.}+e^{i(T-\varepsilon) \beta_{d} / 2}[C G] \begin{array}{l}
1 \\
R_{e}, R_{0}
\end{array}\right\} e^{i \omega t} \\
+\hat{\alpha}_{R_{e}}\left\{[C M]_{R_{e}, R_{0}}^{0}\right\} e^{i \omega t}+\hat{\alpha}_{R_{0}}\left\{[C M] \begin{array}{l}
1 \\
R_{e}, R_{0}
\end{array}\right\} e^{i \omega t}
\end{aligned}
$$

where $\alpha_{R_{e}}$ and $\alpha_{R_{0}}$ are unknown complex displacements of the reference airfoils.

\section{AERODYNAMIC MODEL VERIFICATION}

To verify the finite-cascade unsteady aerodynamic model and influence coefficient technique, predictions from this model are compared to analogous predictions obtained from the uniformly spaced infinite-cascade analys is of Adamczyk and Goldstein (ref. 13). In particular, both analyses are used to predict the transverse-gust unsteady airfojl loading on Verdon's Cascade $B$ flow geometry, depicted schematically in figure 3 . 
interblade phase angle $\beta$ as parameter, are presented in figure 4 . The exce1lent agreement between the two analyses is apparent.

\section{EQUATIONS OF MOTION}

The equations for the single-degree-of-freedom torsional motion of the two reference airfoils of the alternate circumferentially spaced cascade are developed by considering the typical airfoil sections depicted in figure 5 . Positive torsional displacements are defined as a clockwise motion such that the airfoils are in a leading-edge-up configuration. The elastic restoring forces are modeled by linear torsional springs at the elastic-axis location, with the inertial properties of the airfoils represented by their mass moment of inertia about the elastic axis. The equations of motion are then determined by using the Lagrange technique. Only the following two equations of motion are obtained because of the alternate aerodynamic detuning:

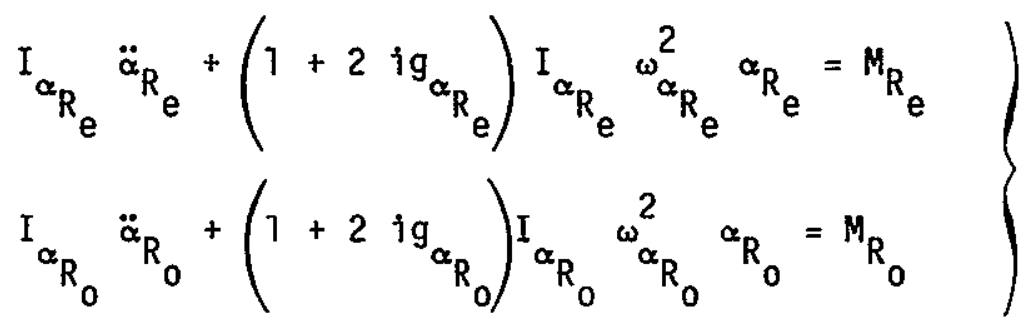

where the undamped natural frequencies are

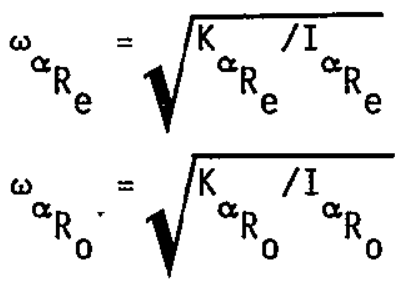

and $g_{\alpha_{R_{e}}}$ and $g_{\alpha_{R_{0}}}$ denote the structural damping coefficients for the

reference airfoils $R_{e}$ and $R_{0}$, respectively.

When the harmonic motion of the reference airfoils is considered and the total unsteady aerodynamic moments defined in equation (11) are used, the equations of motion (eq. 12) can be written in a dimensioniess matrix form:

$$
\left[\begin{array}{cc}
\mu_{e} & {[C M]_{R_{e}}^{1}} \\
{[C M]_{R_{0}}^{0}} & \mu_{0}
\end{array}\right\}\left\{\begin{array}{l}
\hat{\alpha}_{R_{e}} \\
\hat{\alpha}_{R_{0}}
\end{array}\right\}=-\frac{w_{g}}{u_{\infty}}\left\{\begin{array}{cc}
{[C G]_{R_{e}}^{0}+e^{i(1-\varepsilon) \beta_{d} / 2}} & {[C G]_{R_{e}}^{1}} \\
{[C G]_{R_{0}}^{0}+e^{i(1-\varepsilon) \beta_{d} / 2}} & {[C G]_{R_{0}}^{1}}
\end{array}\right\}
$$

where 


$$
\begin{gathered}
\mu_{e}=\mu_{R_{e}} r_{\alpha_{R_{e}}}^{2}+[C M]_{R_{e}}^{0}-\left(1+2 i g_{\alpha_{R_{e}}}\right) \mu_{R_{e}} r_{\alpha_{R_{e}}}^{2} \gamma_{\alpha_{R_{e}}}^{2} \gamma \\
\mu_{0}=\mu_{R_{0}} r_{\alpha_{R_{0}}}^{2}+[C M]_{R_{0}}^{1}-\left(1+2 i g_{\alpha_{R_{0}}}\right) \mu_{R_{0}} r_{\alpha_{R_{0}}}^{2} \gamma_{\alpha_{R_{0}}}^{2} \gamma \\
\mu_{R_{e}, R_{0}}=\frac{m_{R_{e}, R_{0}}}{\pi \rho b^{2}} \\
r_{\alpha_{R_{e}}, R_{0}}^{2}=\frac{{ }_{\alpha_{e}}, R_{0}}{m_{R_{e}, R_{0}} b^{2}} \\
r_{\alpha_{R_{e}}, R_{0}}^{2}=\frac{\omega_{\alpha_{e}, R_{0}}}{\omega_{0}} \\
\gamma=\frac{\omega_{0}^{2}}{\omega^{2}}
\end{gathered}
$$

and where $\omega_{0}$ is the reference frequency.

The aeroelastic forced response of a cascade subjected to an incoming gust can be determined from the solution to equation (13). The resulting displacement vector is given by

$$
\left[\begin{array}{c}
\hat{\alpha}_{R_{e}} \\
\hat{\alpha}_{R_{0}}
\end{array}\right]=-\frac{w_{g}}{u_{\infty}}\left[\begin{array}{cc}
\mu_{e} & {[C M]_{R_{e}}^{]}} \\
{[C M]_{R_{e}}^{0}} & \mu_{0}
\end{array}\right]^{-1}+\left[\begin{array}{cc}
{[C G]_{R_{e}}^{0}+e^{i(7-\varepsilon) \beta_{d} / 2}[C G]_{R_{e}}^{1}} \\
{[C G]_{R_{0}}^{0}+e^{i(7-\varepsilon) \beta_{d} / 2}[C G]_{R_{0}}^{]}}
\end{array}\right]
$$

The mathematical model specified by equation (14) can now be used to determine the forced response of a tuned cascade, an alternate circumferentially spaced aerodynamically detuned cascade, or a structurally deturned cascase. The aerodynamic detuning is accounted for through the influence coefficients $\left[c_{m}^{n}\right]_{R_{e}}, R_{0}$ and $\left[C_{g}^{n}\right]_{R_{e}}, R_{0}$ and the detuning parameter $\varepsilon$. Aiternate blade structural detuning can be incorporated through the ratios $\gamma_{\alpha_{R_{e}}}$ and ${ }^{\gamma_{\alpha_{R_{0}}}}$. 


\section{RESULTS}

To demonstrate the effects of alternate-circumferential-spacing aerodynamic detuning on flow-induced forced response, the single-degree-of-freedom torsion model specified by equation (14) is applied to a 12-bladed rotor. Verdon's Cascade B flow geometry, schematically depicted in figure 3 , is used as a uniformly spaced baseline cascade configuration. Cascade $B$ is a standard cascade for flutter calculations. As forced response, not flutter, is of interest in this report, the baseline cascade must be stable, that is, flutter free, for all interblade phase angles. This was accomplished by maintaining the geometric cascade parameters and slightly adjusting the inlet Mach number and the reduced frequency values from those of verdon.

For a uniformly spaced cascade, the eigenvalues and corresponding eigenvectors of equation (13) represent traveling wave modes which have interblade phase angles of $\beta$ and $\beta+\pi$. For the aerodynamically detuned cascade, the traveling wave modes have interblade phase angles of $\beta_{d}$ and $\beta_{d}+\pi$, where $\beta_{d}=2 \beta$. For example, for $\beta_{d}=180^{\circ}$ and a tuned cascade, the two eigenvalues correspond to traveling wave modes with interblade phase angles of $\beta= \pm 90^{\circ}$. These same two eigenvalues would also be obtained for $\beta_{d}=-180^{\circ}$.

The root locus plot of the eigenvalues for the baseline cascade $B$ configuration is presented in figure 6 , with these eigenvalues expressed as

$$
i\left(\omega / \omega_{0}\right)=u+i v
$$

where $u$ is a measure of the total system damping, and $v$ is the damped natural frequency of the airfoils. In this study, the structural damping coefficients $g_{\alpha_{R_{e}}}$ and $g_{\alpha_{R_{0}}}$ are taken to zero. Thus, the system damping is due only to the motion-dependent unsteady aerodynamic loading.

The effect of aerodynamic detuning on the forced response of cascade $B$ subjected to gust loads characterized by interblade phase angles of $\beta=0^{\circ}$, $30^{\circ}, 90^{\circ}$, and $-90^{\circ}$ ( $\mathrm{fig} .6$ ) was investigated. These correspond to detuned interblade phase angles of $\beta_{\alpha}=0^{\circ}, 60^{\circ}, 180^{\circ}$, and $-180^{\circ}$. These interblade phase angles were selected so that the gust loads would excite modes of the airfoils that exhibit both low and high total system damping, as determined by the real parts of the cascade eigenvalues.

The baseline Cascade $B$ configuration, $\varepsilon=0$ percent, is aerodynamically detuned by alternating the circumferential airfoil spacing by an amount $\varepsilon=10$ percent and $\varepsilon=17$ percent. Figure 7 schematicaliy depicts the Cascade $B$ flow structure for the baseline and a detuned configuration and demonstrates that aerodynamic detuning results in different unsteady aerodynamic loadings on the surfaces of the two reference airfoils. The variation in the eigenvalues for the baseline and detuned Cascade B configuration as a function of the level of aerodynamic detuning for the interblade phase angles of interest is presented in table $I$.

The effect of aerodynamic detuning on the amplitude of response of the reference airfoils $R_{e}$ and $R_{0}$ as a function of the nondimensional gust frequency $\omega / \omega_{0}$ is shown in figures 8 to 11 . The gust frequency is norma $1-$ ized with respect to the reference frequency $\omega_{0}$, which represents the undamped 
torsional frequency of the airfoils. Also, the amplitude of response $\bar{\alpha}$ is nondimensionalized with the maximum response amplitude obtained for the baseline tuned configuration $\bar{\alpha}_{0}$.

For a gust with a forward traveling wave characterized by $\beta_{d}=180^{\circ}$, the effect of aerodynamic detuning on the amplitude of response of the reference airfoils is presented in figure 8 . Aerodynamic detuning results in a significant decrease in the maximum amplitude of response; the greatest decrease is associated with the highest level of detuning. For $\varepsilon=10$ percent, the maximum amplitude of response is decreased of the order of 50 percent, whereas with $\varepsilon=17$ percent, this decrease is approximately 60 percent. In this case, aerodynamic detuning is of significant benefit with regard to passive control of forced response.

Figure 8 also demonstrates that the two reference airfoils of the detuned cascade do not have the same response amplitudes. This effect becomes more pronounced as the level of aerodynamic detuning is increased. It is associated with the differences in the chordwise distribution of the unsteady aerodynamic loading on the surfaces of the two reference airfoils due to the nonuniform airfoil spacing of the aerodynamically detuned cascades, as was depicted in figure 7 .

Figure 9 presents the effect of aerodynamic detuning on Cascade $B$ when it is subjected to a gust load with a forward traveling wave with $B_{d}=60^{\circ}$. As aerodynamic detuning is introduced, the maximum response amplitude of the reference airfoils decreases, with the largest decrease associated with the higher level of detuning. For $\varepsilon=17$ percent, the maximum response amplitude of airfoil $R_{e}$ decreased of the order of 30 percent, while that of airfoil $R_{0}$ decreased approximate $7 y$ percent. Thus, aerodynamic detuning is also very beneficial in this case.

For a gust load with an interblade phase angle of $\beta_{d}=0^{\circ}$, figure 10 shows the normalized amplitude of response of the two reference airfoils with the level of aerodynamic detuning as a parameter. The maximum response of both reference airfoils is obtained for the uniformly spaced baseline cascade, $\varepsilon=0$ percent, at about $\omega / \omega_{0}=1.015$. Aerodynamic detuning decreases the maximum response amplitude of the reference airfoils. In this case, however, the decrease in the response is smal1. As the level of detuning is increased to 17 percent, both reference airfoils exhibit a small response near $\omega / \omega_{0}=0.99$. This type of response could be due to the aerodynamic coupling which exists between the odd- and even-numbered airfoils.

For a gust with a backward traveling wave characterized by $\beta_{d}=-180^{\circ}$, the effect of aerodynamic detuning on the amplitude of response of the reference airfoils is presented in figure 11. Both reference airfoils exhibit resonant behavior in the vicinity of the frequency ratios $\omega / \omega_{0}=0.96$ and 1.06. The eigenvectors indicate that the cascade can vibrate in either of two mode shapes, one with $\beta_{d}=+780^{\circ}$ and the other with $\beta_{d}=-180^{\circ}$. For the baseline tuned cascade, the maximum blade response is in the mode with $\beta_{d}=-180^{\circ}$, which has a resonant frequency at $\omega / \omega_{0}=1.056$, as indicated in table $I$. The baseline tuned cascade exhibits very little response in the mode associated with $\beta_{d}=780^{\circ}$. However, as the level of detuning is increased, the two modes become coupled, and, even though the cascade is being excited by a gust load with $\beta_{d}=-180^{\circ}$, it has significant response in the mode associated with 
$\beta_{d}=180^{\circ}$. Thus, although the introduction of aerodynamic detuning decreased the maximum response amplitude of the baseline casecade near $\omega / \omega_{0}=1.06$, it significantly increased amplitudes of response near $\omega / \omega_{0}=0.96$.

\section{SUMMARY OF RESULTS}

An aeroelastic model was developed to investigate the effect of alternatecircumferential-spacing aerodynamic detuning on the supersonic-flow-induced forced response of a turbomachine blade row. The model of the unsteady aerodynamic loading on the blading is determined from a finite cascade analysis in conjunction with an influence-coeffjcjent technique. This technique makes it possible to consider various gust loadings without having to perform a complete unsteady aerodynamic analysis for each interblade phase angle. The unsteady aerodynamic loading is considered as a transverse gust convected with the through flow past a stationary airfoll cascade as well as the motion-induced unsteady loading which results when the cascade harmonically responds to this gust. The aeroelastic model which was developed in this report was used to analyze both uniformly spaced tuned and aerodynamically detuned cascade configurations. However, the method is not limited to these configurations since it could be applied to a structurally detuned cascade as well.

This aeroelastic model was used to investigate the effect of aerodynamic detuning on the flow-induced forced response of a 12-bladed rotor, with verdon's Cascade B flow geometry as a uniformly spaced baseline configuration.

For a forward traveling gust load, aerodynamic detuning generally had a very beneficial effect on the maximum airfoil response. This resulted in significantly decreased maximum amplitudes of response for many interblade phase angles. However, for backward-traveling gust loads, aerodynamic detuning was not as beneficial, resulting in relatively small decreases in maximum response amplitudes. In addition, with a backward-traveling gust load with an interblade phase angle $\beta_{d}=-180^{\circ}$, aerodynamic detuning resulted in a coupling of response modes, with the coupled mode exhibiting a significantly increased response amplitude.

Aerodynamic detuning has potential benefit as a passive control mechanism for the flow-induced forced response of a blade row, particularly for forwardtraveling gust loads. However, for some gust load interblade phase angles, aerodynamic detuning may result in no decrease or even an increase in the amplitudes of response.

\section{REFERENCES}

1. Whitehead, D.S., "Effect of Mistuning on the Vibration of Turbomachine Blades Induced by Wakes," Journal of Mechanical Engineering Science, Vo1. 8, No. 1, Mar. 1966, pp. 15-21.

2. Kaza, K.R.V. and Kielb, R.E., "Flutter and Response of a Mistuned Cascade in Incompressible flow," AIAA Journal, Vol. 20, No. 8, Aug. 1982, pp. 1120-1127. 
3. Kielb, R.E. and Kaza, K.R.V., "Aeroelastic Characteristics of a Cascade of Mistuned Blades in Subsonic and Supersonic Flows, "Journal of Vibration, Stress and Reliability in Design, Vol. 105, No. 4, Oct. 1983, pp. 425-433.

4. Bendiksen, 0.0., "Flutter of Mistuned Turbomachinery Rotors," Journal of Engineering for Gas Turbines and Power, Vol. 106, No. 1, Jan. 1984, pp. 25-33.

5. Crawley, E.F. and Ha 11, K.C., "Optimization and Mechanisms of Mistuning in Cascades," Journal of Engineering for Gas Turbines and Power, V07. 107, No. 2, Apr. 7985 , pp. 478-426.

6. Hoyniak, D. and Fleeter, S., "Aerodynamic' Detuning Analysis of an Unstalled Supersonic Turbofan Cascade," ASME Paper 85-GT-192, July 1985.

7. Verdon, J.M. and McCune, J.E., "Unsteady Supersonic Cascade in Supersonic Axial Flow," AIAA Journal, Vol. 13, No. 2, Feb. 1975, pp. 193-201.

8. Verdon, J.M., "The Unsteady Aerodynamics of a Finite Supersonic Cascade with Subsonic Axial Flow," Journal of Applied Mechanics, Vol. 40, No. 3, Sept. 1973, pp. 667-671.

9. Brix, C.W. and Platzer, M.F., "Theoretical Investigation of Supersonic Flow Past Oscillating Cascades with Subsonic Leading Edge Locus," AIAA Paper No. 74-74, Jan. 1974.

10. Caruthers, J.E. and Riffel, R.E., "Aerodynamic Analysis of a Supersonic Cascade Vibrating in a Complex Mode," Journal of Sound and Vibration, Vo1. 71 , No. 2, July 22, 1980, pp. 171 -183 .

11. Nagashima, T. and Whitehead, D.S., "Linearized Supersonic Unsteady Flow in Cascades," ARC-R\&M-3811, Aeronautics Research Counci1, London, England, 1977.

12. Goldstein, M.E., Aeroacoustics, McGraw Hill, New York, 1976.

13. Adamczyk, J.J. and Goldstein, M.E., "Unsteady Flow in a Supersonic Cascade with Subsonic Leading Edge Locus," AIAA Journal, Vol. 16, No. 12, Dec. 1978, pp. 1248-1254. 
TABLE I. - CASCADE EIGENVALUES

\begin{tabular}{|c|c|c|c|}
\hline $\begin{array}{c}\text { Interblade } \\
\text { phase angle, } \\
\beta_{d}, \\
\text { deg }\end{array}$ & $\begin{array}{c}\text { Aerodynamic } \\
\text { detuning, } \\
\varepsilon, \\
\text { percent }\end{array}$ & Real $\left(i \omega / \omega_{0}\right)$ & Imaginary $\left(i \omega / \omega_{0}\right)$ \\
\hline 0 & 0 & $-0.7479 \times 10^{-2}$ & 1.015 \\
& 10 & $-.7144 \times 10^{-2}$ & 1.015 \\
60 & 17 & $-.6734 \times 10^{-2}$ & 1.015 \\
& 0 & $-0.2320 \times 10^{-2}$ & 0.9916 \\
& 10 & $-.2707 \times 10^{-2}$ & .9923 \\
180 & 17 & $-.3533 \times 10^{-2}$ & .9911 \\
& 0 & $-0.1545 \times 10^{-2}$ & 0.9649 \\
& 10 & $-.3110 \times 10^{-2}$ & .9649 \\
-180 & 17 & $-.4334 \times 10^{-2}$ & .9625 \\
& 0 & $-3.234 \times 10^{-2}$ & 1.056 \\
& 10 & $-3.318 \times 10^{-2}$ & 1.053 \\
& 17 & $-3.023 \times 10^{-2}$ & 1.050 \\
\hline
\end{tabular}




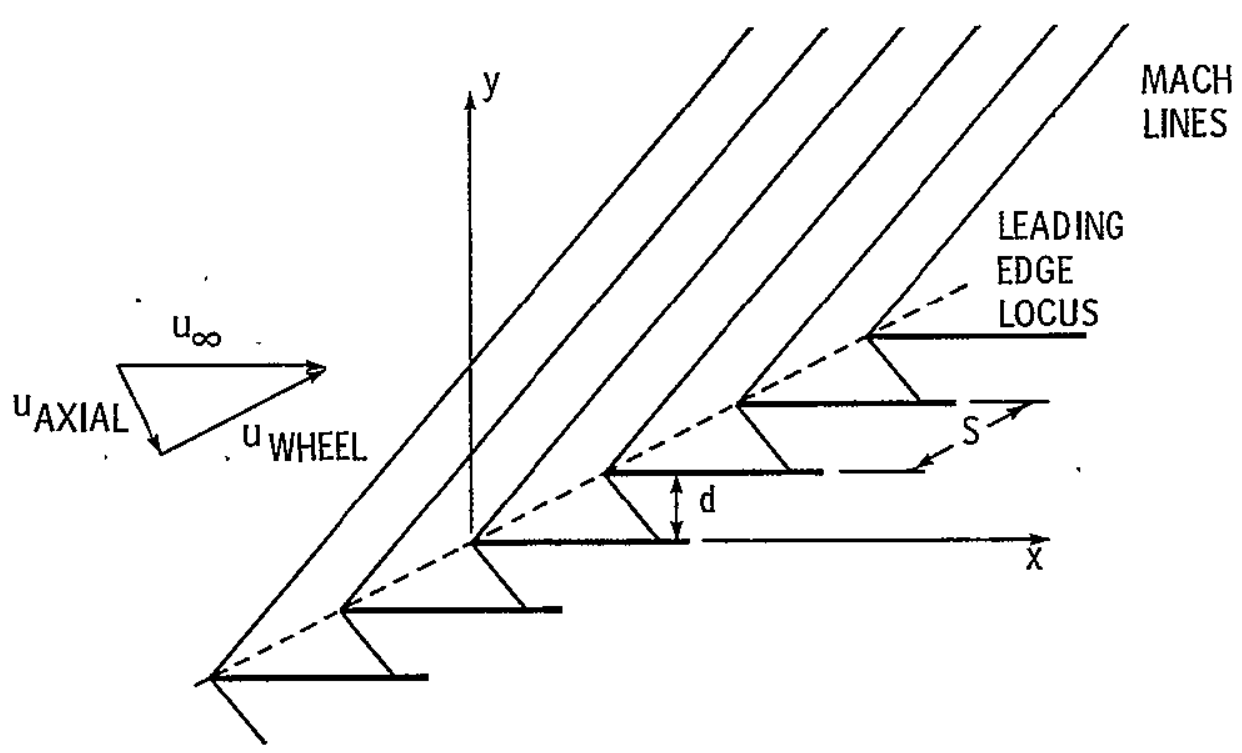

Figure 1. - Flat-plate airfoil cascade in supersonic with subsonic axial component inlet flow field.

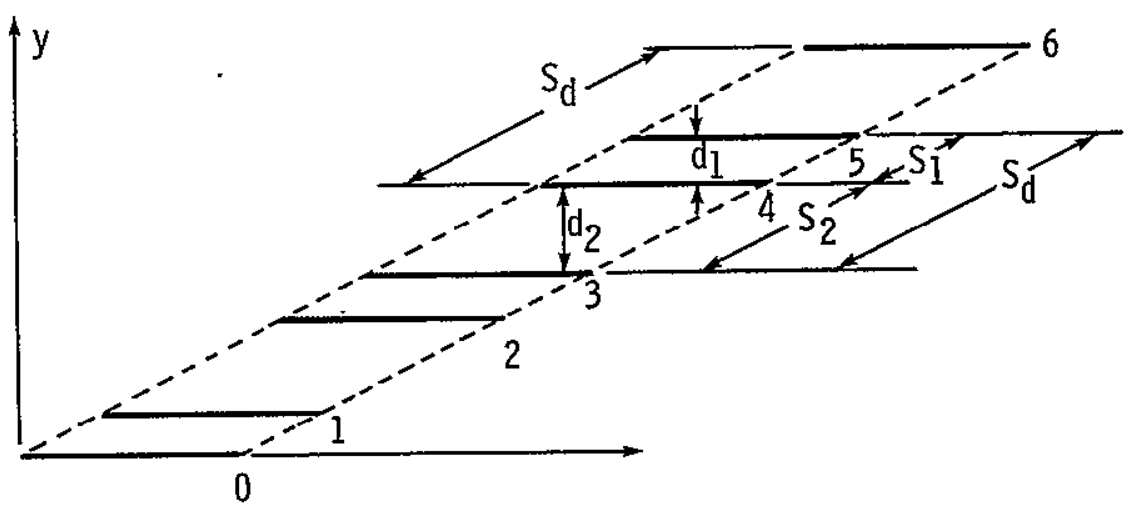

Figure 2 - Finite cascade representation for Aerodynamic detuning by alternate nonuniform circumferential spacing.

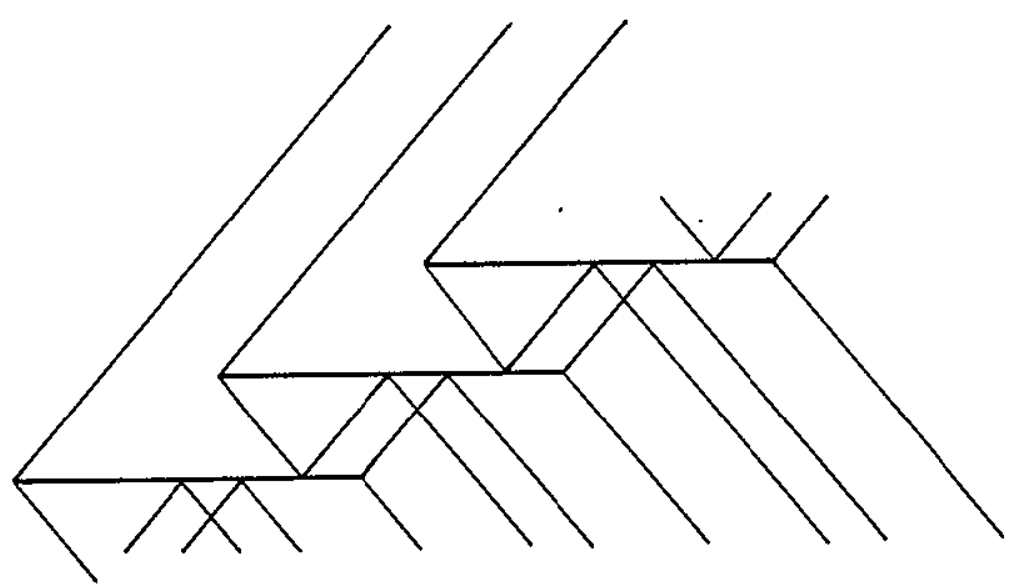

Figure 3. - Cascade B flow geometry. 


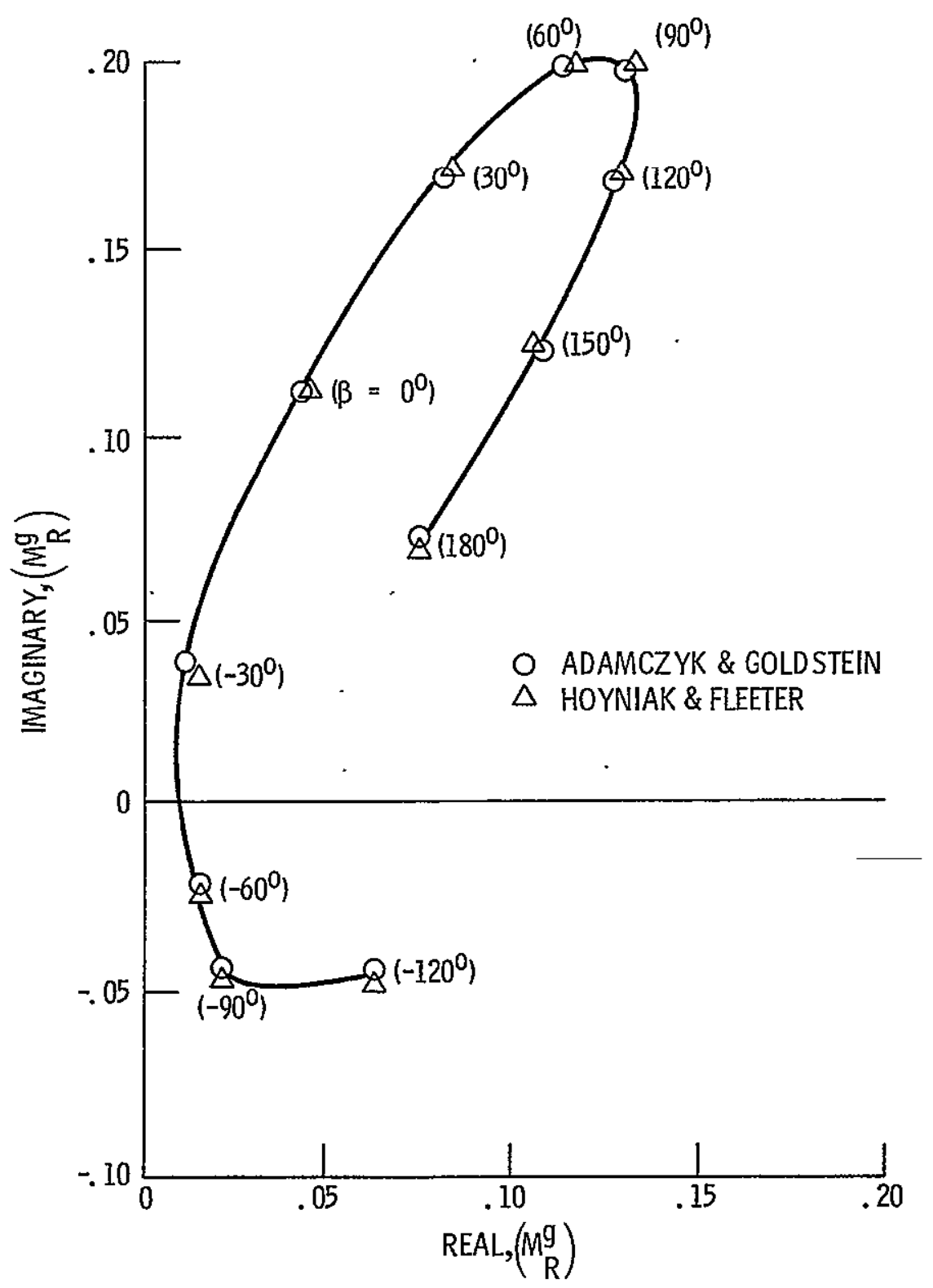

Figure 4. - Comparison of finite cascade influence-coefficient model with infinite cascade analysis for gust, $M=1.281$; $k=1.00$. 

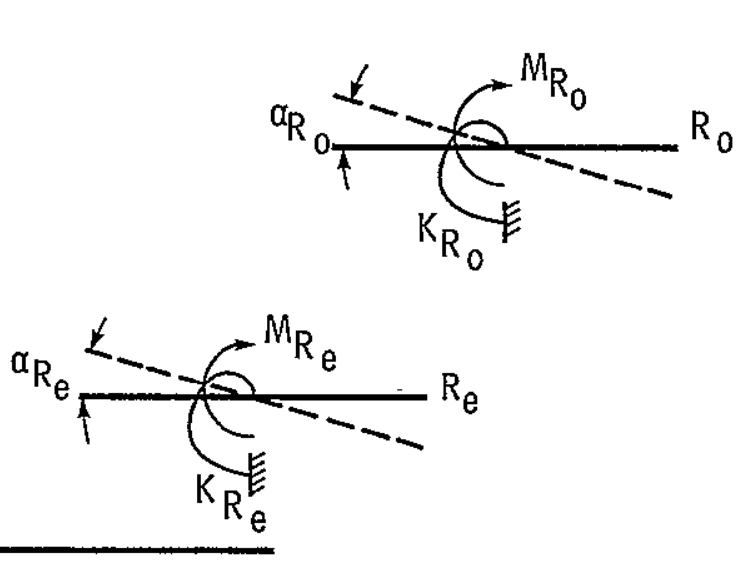

Figure 5. - Single-degree-of-freedom model for aerodynamically detuned cascade.

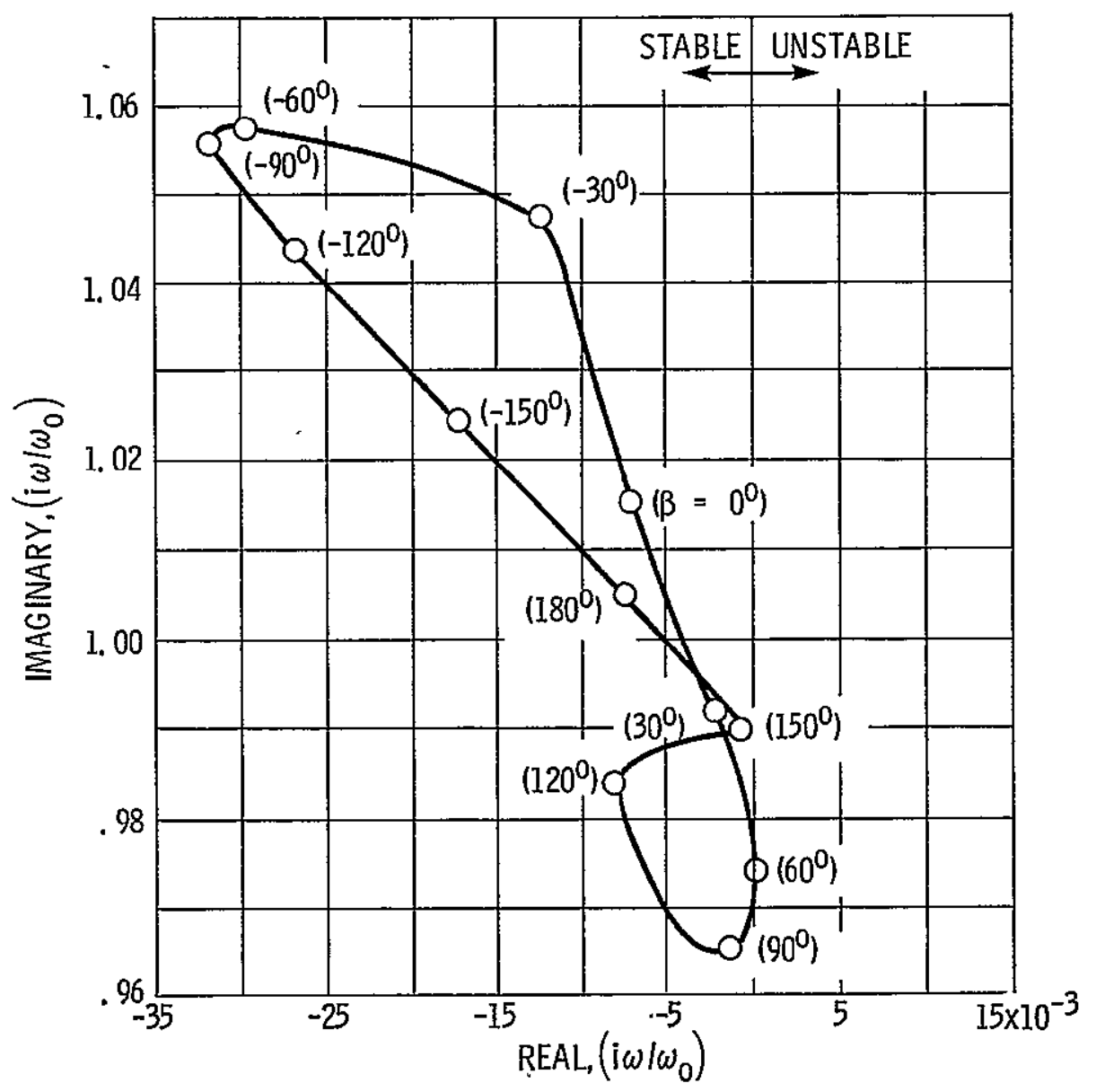

Figure 6. - Stability of baseline Cascade B configuration. $M=1.150 ; k=1.00$. 


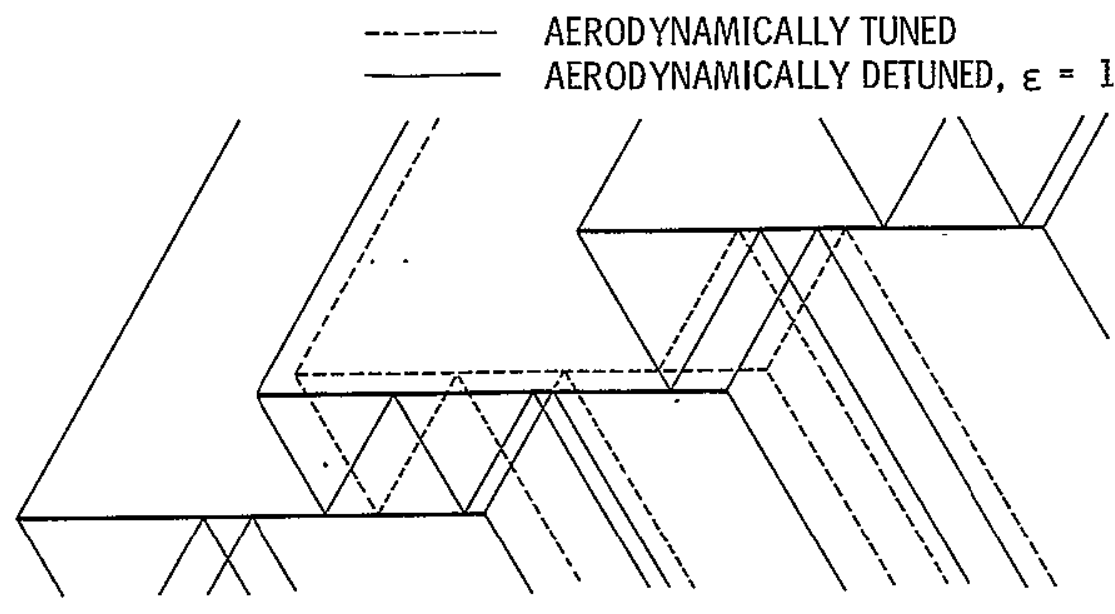

Figure 7. - Baseline and aerodynamically detuned cascade flow geometries. $M=1.15 ; k=1.00$.

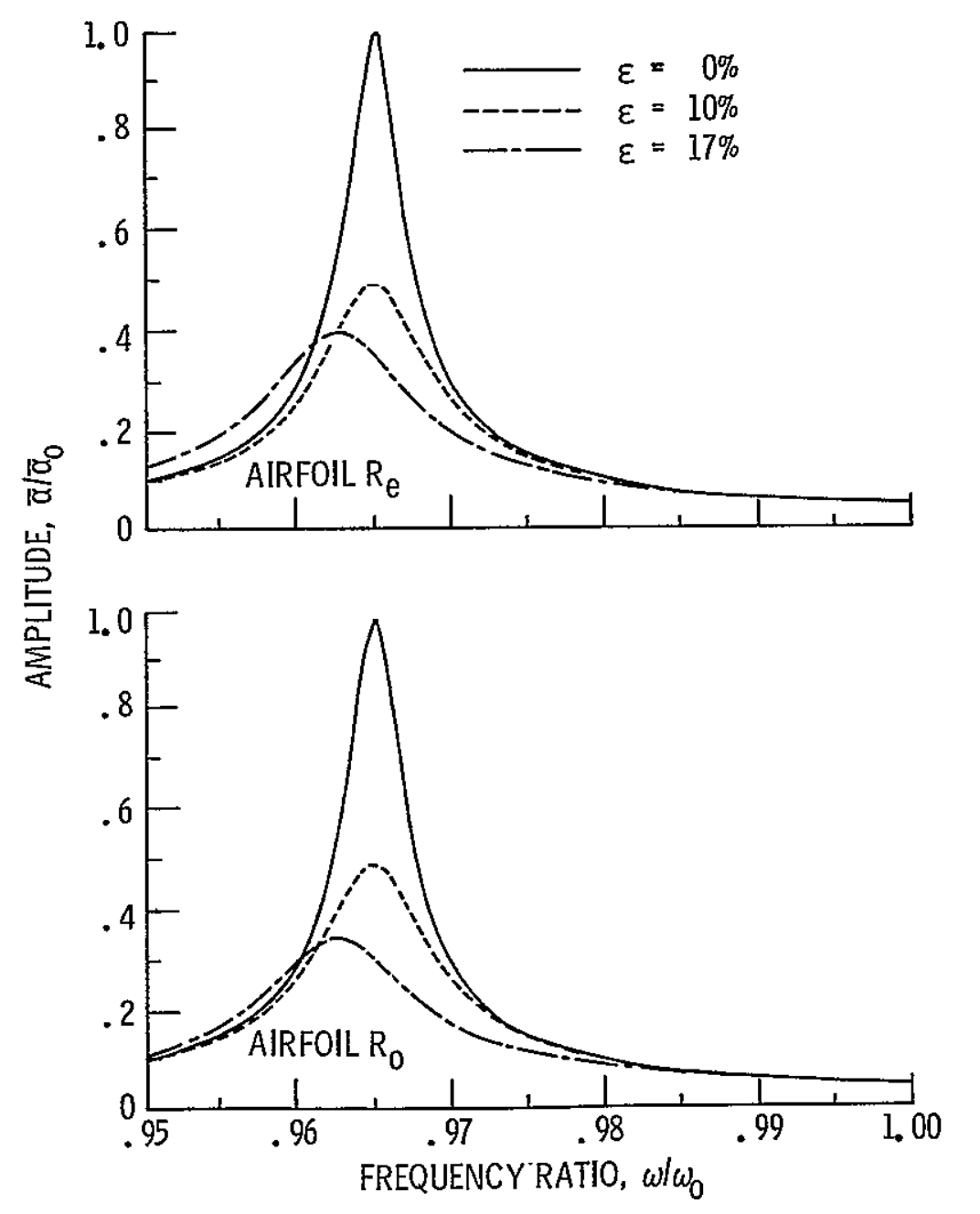

Figure 8. - Effect of aerodynamic detuning on airfoil response of cascade $B$ for gust with $\beta_{d}=180^{\circ}$. $M=1.150 ; k=1.00$. 
ORIGINAZ PAEE IS

OF POOR QJALTY

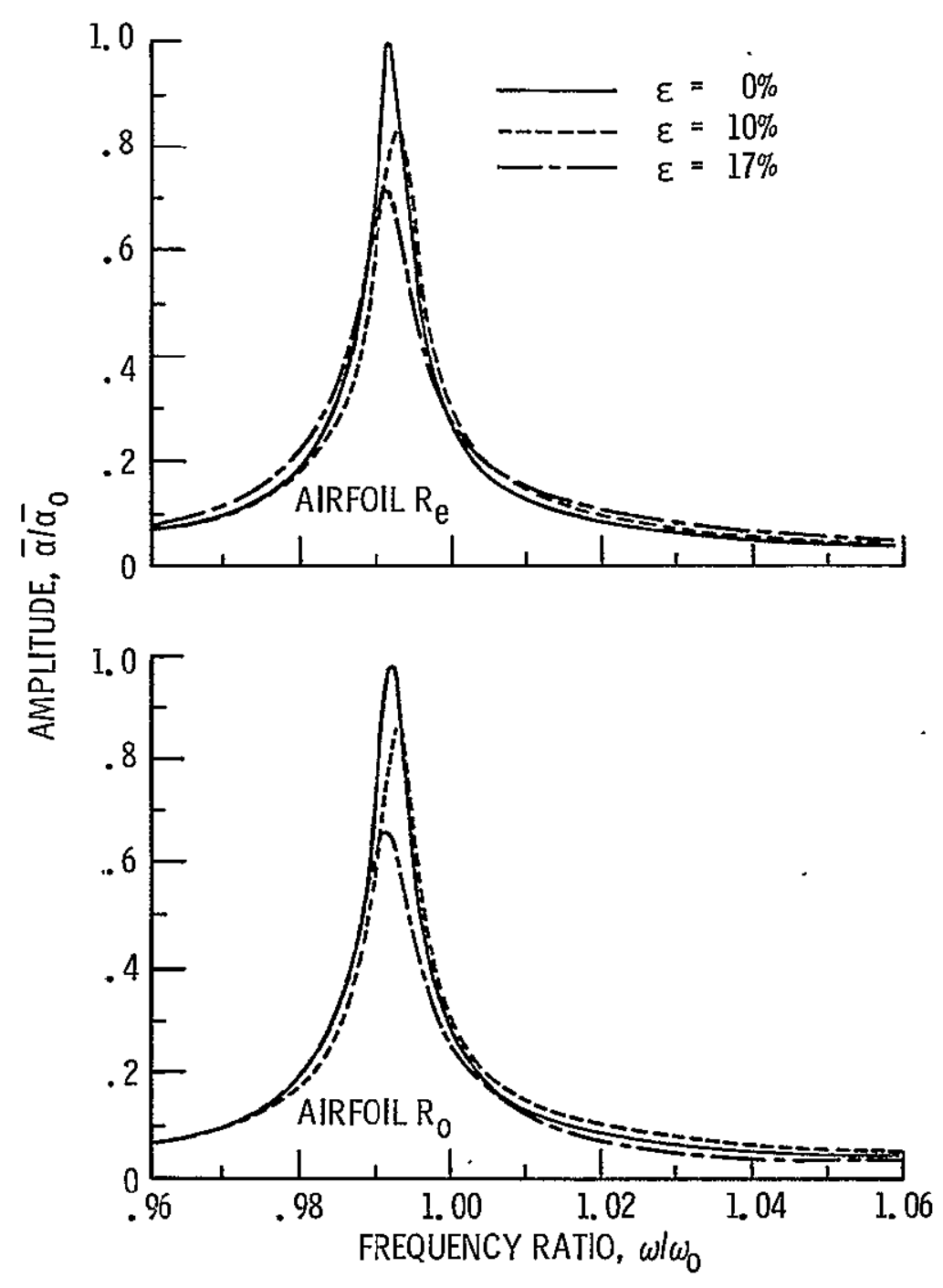

Figure 9. - Effect of aerodynamic detuning on airfoil response of cascade $B$ for gust with $\beta_{d}=60^{\circ}$. $M=1.150 ; k=1.00$. 


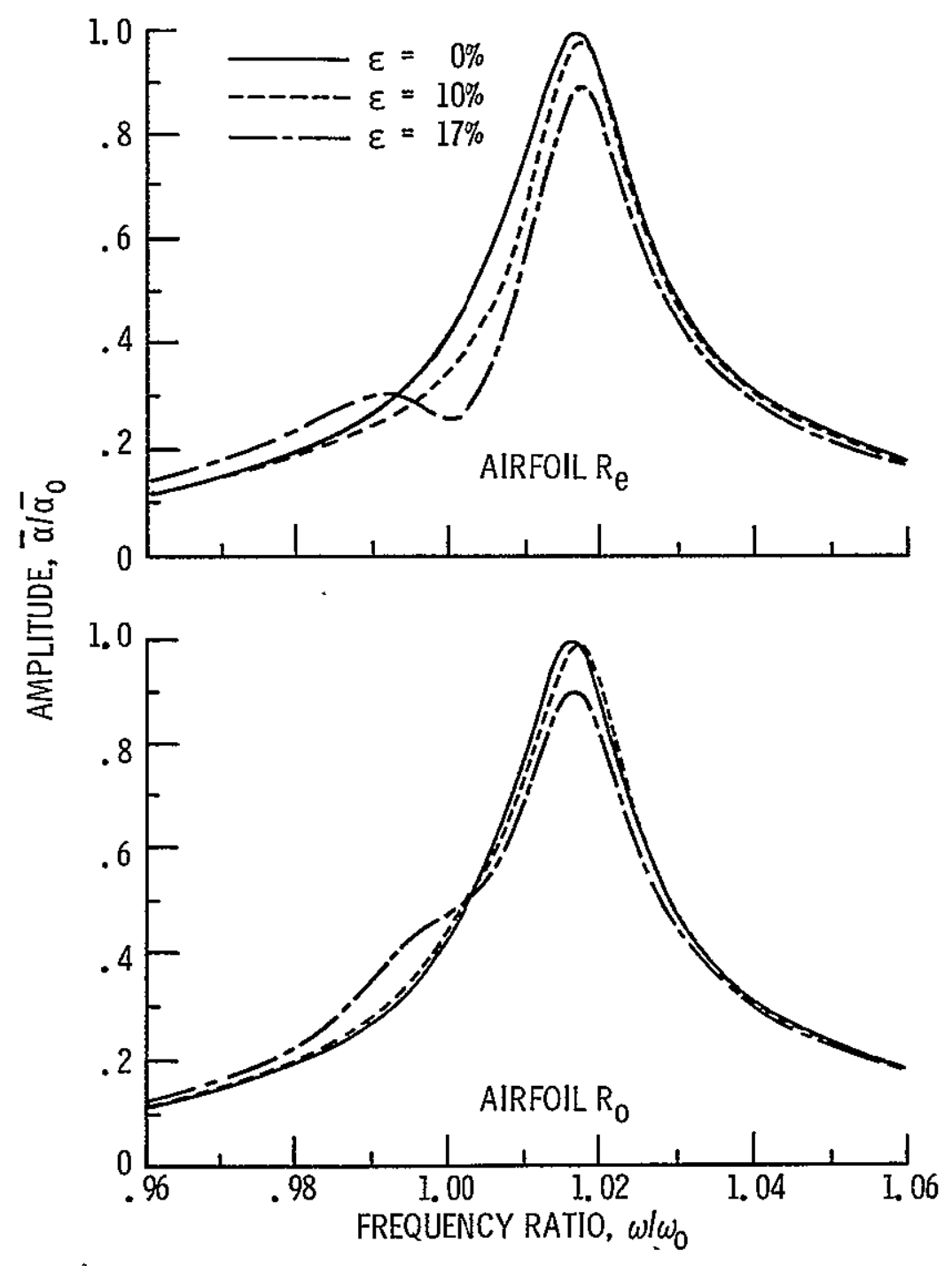

Figure 10. - Effect of erodynamic detuning on airfoil response of cascade $B$ for gust with $\beta_{d}=0^{0} . M=1.150$; $\mathrm{k}=1.00$. 


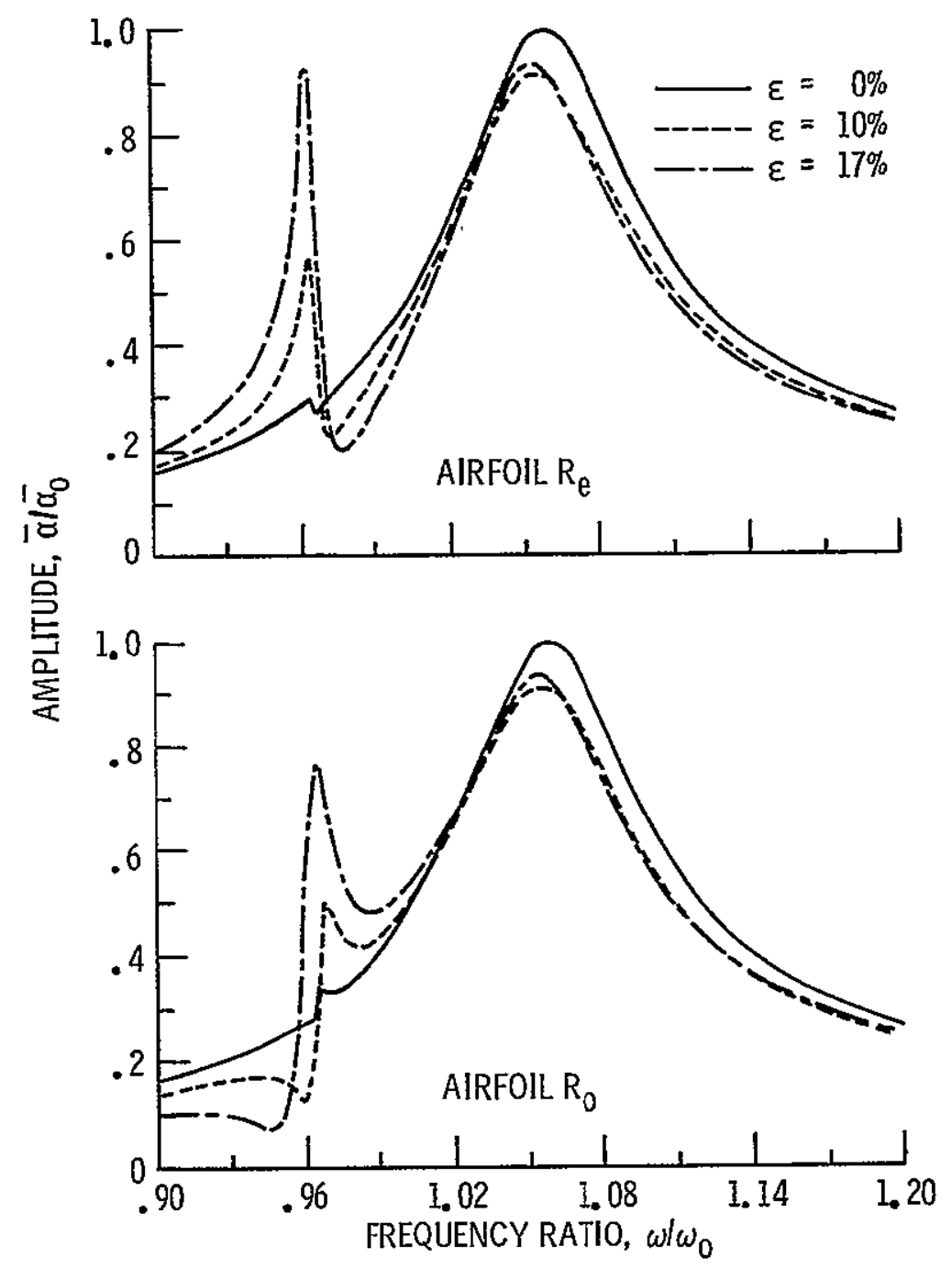

Figure 11. - Effect of aerodynamic detuning on airfoil response of cascade $B$ for gust with $\beta_{d}=180^{\circ}$. $M=1.150 ; k=1.00$. 


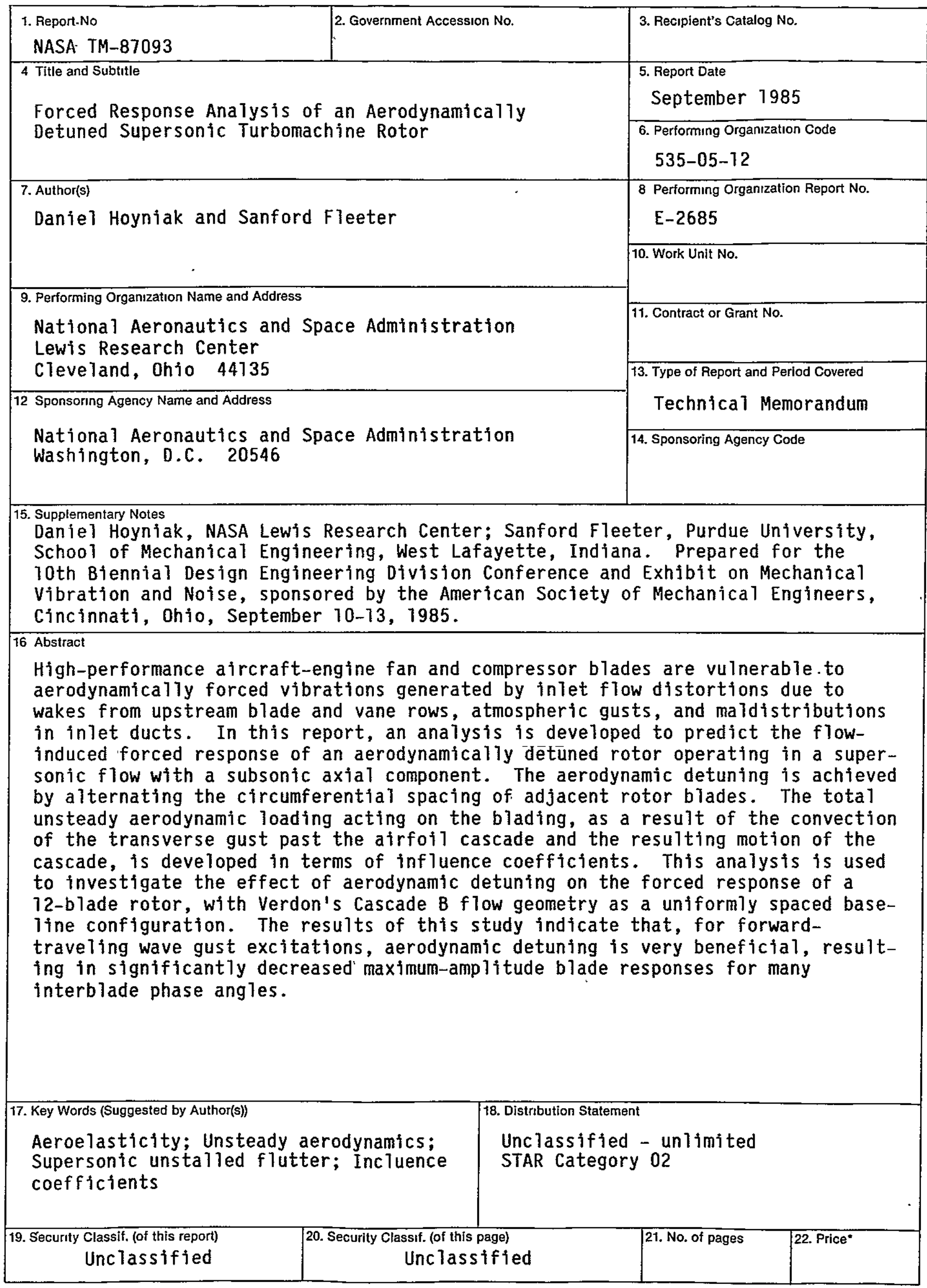

${ }^{\star}$ For sale by the National Technical Information Service, Sprıngfıeld, Vırginia 22161 
Natıonal Aeronautıcs and Space Admınıstration

Lewis Research Center

Cleveland, Ohıo 44135

Official Business

Penalty for Private Uso $\$ 300$
SECOND CLASS MAIL

ADDRESS CORRECTION REQUESTED

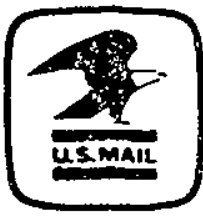

Postage and Fees Paic National Aeronautics ar Space Administration NASA-451 